\title{
Effect Evaluation of Biomedical Experiment Teaching Based on Intelligent Sensor
}

\author{
Zhicong Ma \\ Department of Medical College, Xuchang University, Henan Xuchang 461000, China \\ Correspondence should be addressed to Zhicong Ma; 12014131@xcu.edu.cn
}

Received 11 November 2021; Revised 5 January 2022; Accepted 22 January 2022; Published 14 February 2022

Academic Editor: Gengxin Sun

Copyright (C) 2022 Zhicong Ma. This is an open access article distributed under the Creative Commons Attribution License, which permits unrestricted use, distribution, and reproduction in any medium, provided the original work is properly cited.

\begin{abstract}
With the continuous development and progress of nanotechnology, its biosensors have been widely used in biomedical experimental teaching, and good experimental results have been achieved. Graphene, as a new nanomaterial with large surface area, good thermal conductivity, and unique electrical conductivity, has unique advantages in the field of biosensor preparation. Based on this, this paper will prepare the electrochemical sensor applied to biomedical experimental teaching based on graphene, optimize the detection sensitivity and detection range of graphene electrochemical sensor based on the corresponding experimental conditions, and improve its corresponding stability and reusability. At the level of electrochemical activity of biosensors, this paper innovatively uses the electric AC impedance method to detect the electrochemical activity, so as to accurately evaluate the key characteristics of biosensors. Based on the preparation of biosensors and the results of biological experiments, this paper will design a network-based biomedical experiment teaching effect evaluation system, and realize the basic functions of teacher-student interaction, teaching effect evaluation, sensor performance evaluation and so on. Based on the above, the electrochemical sensor based on graphene and a conductive polymer solution is actually prepared in this paper. At the same time, the electrocatalysis experiment is carried out based on the sensor, and the experimental teaching effect is systematically evaluated. The experimental results show that the sensitivity of the biosensor proposed in this paper is increased by about 10\% compared with the traditional biosensor, the corresponding preparation complexity is reduced by nearly $1 / 3$, and the corresponding reusability is increased by $30 \%$. Therefore, the biomedical experiment teaching effect evaluation system proposed in this paper has good evaluation effect. It can provide accurate reference for the evaluation of biological experiment teaching effect, so it has important value and significance.
\end{abstract}

\section{Introduction}

As an important means of modern biomedical experiments, the biosensor is essentially a high-precision biosensor formed by combining the sensitive parts of organisms and their derivatives through physical and chemical energies. It linearizes the intensity of the detected biological signal with the physicochemical characteristics of the detected related organisms and converts it into a digital signal for processing $[1,2]$. The cell biosensor is mainly composed of two parts: a molecular factor recognition processor and an energy converter, in which the corresponding energy converter is the main core module, which essentially converts biological signals into electrical signals, optical signals, thermal signals, and acoustic signals. In addition, molecular factors commonly used in biomedical experimental teaching include the detected organisms, tissues, nucleic acids, and organelles and corresponding biological derived materials [3-5]. The preparation technology of biosensors includes the crossintegration of biology, physics, chemistry, and other disciplines. Therefore, the corresponding biosensors in conventional biomedical experiments mainly include sound sensors, optical sensors, electrical sensors, semiconductor sensors, and electrochemical sensors [6-8]. As the most commonly used biosensor in biomedical experimental teaching, electrochemical sensors are mainly divided into cell tissue sensors, cell sensors, RNA sensors, microbial sensors, and enzyme sensors $[9,10]$. Electrochemical sensors 
often have the advantages of high sensitivity, fast response, convenient operation, and miniaturization and integration in biological teaching experiments. Therefore, it is of obvious value to study the sensor and its effect in biomedical experimental teaching $[11,12]$.

As an important biological material for biosensors, nanomaterials have unique biological detection advantages due to their quantum size effect, surface effect, small size effect, and macroquantum tunnel effect [13-15]. Graphene, as a new material for preparing biosensors in nanomaterials, has high charge carrier mobility [16]. Due to its large surface area, high electrochemical activity, and high electron mobility, it has obvious structural advantages as an electrochemical biosensor. A conventional graphene electrochemical biosensor can detect the dose of hydrogen peroxide with high sensitivity, so as to fix the electrochemical behavior of egg white matter [17]. The detection of biological glucose can be realized by using the electron migration of graphene derivatives between the electrode substrate and the redox active center of glucose. The detection of dopamine similar to a nerve substance can be realized based on microwave plasma enhancement technology, and the biosensor technology based on graphene can accurately detect the low concentration of dopamine in the biological system. High concentrations of interfering substances are eliminated in a wide range. Therefore, compared with traditional sensors, graphene-based biosensor technology can achieve highprecision detection of dopamine and other nerve substances and achieve good biomedical teaching experimental results $[18,19]$. In the corresponding biomedical experiment teaching of deoxyribonucleotide, the detection of deoxyribonucleotide is very important and meaningful. The corresponding biomedical experiment teaching effect also directly affects the learning effect of students and reflects the teaching quality of teachers. Based on this, the excellent electrochemical characteristics of the graphene biosensor can detect with high precision deoxyribonucleotides and double-stranded deoxyribonucleotides. Graphene biosensors can provide ultrahigh-density active edge sites [20].

In view of the above corresponding research status and existing problems, this paper focuses on the preparation technology and application of the biosensor based on graphene and systematically gives the design and implementation of the biomedical experiment effect evaluation system and experimental verification. The specific research details are as follows: the electrochemical sensor applied to biomedical experiment teaching based on graphene is prepared and verified to optimize the detection sensitivity and detection range of the graphene electrochemical sensor under the corresponding experimental conditions and improve its corresponding stability and reusability. At the level of the electrochemical activity of the biosensor, this paper innovatively uses the electric AC impedance method to detect the electrochemical activity, so as to accurately evaluate the key characteristics of the biosensor, based on the preparation and production of the biosensor. The advantages of the corresponding electrochemical analysis method are as follows: high sensitivity. The lowest detection limit is $10-12 \mathrm{~mol} / \mathrm{L}$. High accuracy: for example, coulometric analysis and elec- trolytic analysis have high accuracy. The former is especially suitable for the determination of trace components, and the latter is suitable for the determination of high content components. Wide measuring range: potentiometric analysis and microcoulometric analysis can be used for the determination of trace components. Electrolytic analysis, capacitance analysis, and Coulomb analysis can be used for the analysis of medium content components and pure substances. The instrument and equipment are simple, the price is low, the debugging and operation of the instrument are simple, and it is easy to realize automation. Poor selectivity: the selectivity of electrochemical analysis is generally poor, but the selectivity of the ion selective electrode method, polarography, and controlled cathodic potential electrolysis method is high. According to the different electrical quantities measured, electrochemical analysis methods can be divided into conductivity analysis, potential analysis, voltammetry and polarography, and electrolysis and coulometry. Based on the above, an electrochemical sensor based on graphene and the conductive polymer solution is actually prepared and tested based on the sensor electrocatalysis experiment, and the experimental teaching effect is systematically evaluated. The experimental results show that the biosensor proposed in this paper has the advantages of high sensitivity, simple preparation, and high availability. At the same time, it also further verifies that the biomedical experimental teaching effect evaluation system proposed in this paper has good evaluation effect and can provide accurate information for the evaluation of biological experimental teaching effect reference resources.

The structure of this paper is arranged as follows: the second section of the article will analyze and study the preparation technology of the biosensor and the research status of biomedical teaching experimental effect evaluation system; the third section of this paper will focus on the preparation technology and optimization process of the graphene biosensor and give the design process of the biomedical teaching experimental effect evaluation system; the fourth section is mainly the validation experiment and analysis; finally, this paper will be summarized.

\section{Correlation Analysis: Analysis of the Research Status of Sensor-Based Biomedical Experimental Teaching}

At present, the research on biomedical experiment teaching based on sensors mainly focuses on the preparation technology of the biosensor and the evaluation of the teaching effect. At the level of biosensor preparation, a large number of researchers and research institutions have studied and analyzed it. Relevant researchers in Asia have prepared a graphene-based bioelectrochemical sensor based on host guest supramolecular interaction and achieved certain experimental results, At the same time, at the level of graphene biosensor preparation, relevant Japanese scientists have realized high-precision detection of hydrogen peroxide based on the graphene biosensor modified by gold nanoparticles. This detection technology has simplified the detection 


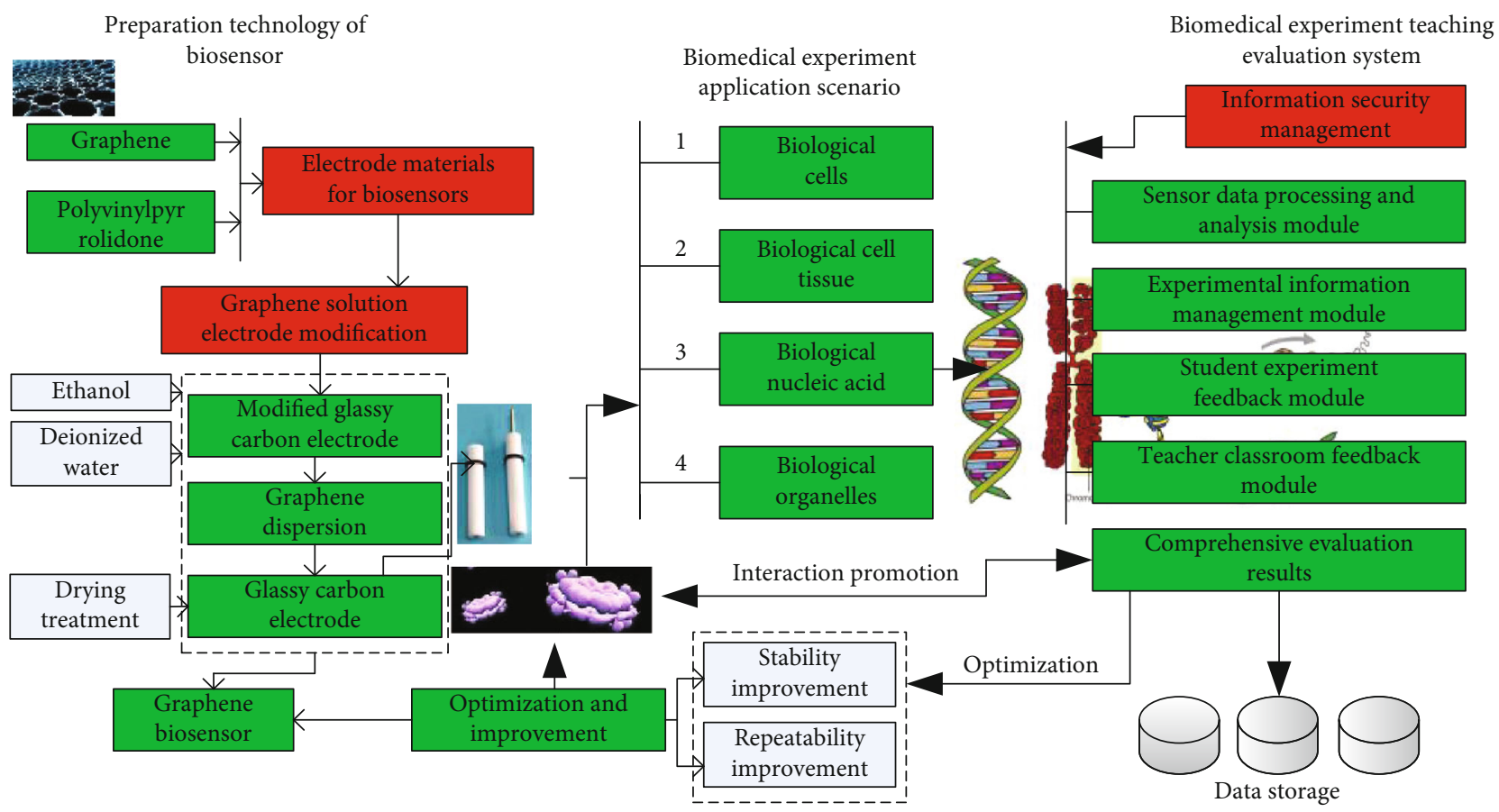

FIGURE 1: Principle block diagram of biomedical teaching evaluation system based on biosensor.

and has certain popularization value $[21,22]$. Relevant European scientists have modified the glassy carbon electrode by the interaction of gold nanoparticles and graphene, so as to realize the high-precision detection of hydrogen peroxide. At the same time, the biosensor has a highly flexible detection response and experimental repeatability [23]. Based on the combination of gold nanoparticles and graphene, relevant scientific institutions in the United States have improved it. It uses gold nanoparticle clusters to modify the corresponding electrode, so as to realize the preparation of a semiamino acid biosensor. This biosensor successfully improves the electron migration rate on the electrode surface, greatly improves the sensitivity of the sensor, and further improves the detection range [24]. Relevant Chinese scientists have also conducted a lot of research on the preparation of biosensors. Relevant research mainly uses the selfassembly technology of gold nanoparticles and plasma polymer deposition technology to prepare immunosensors. The biosensor constructed based on this technology can improve the biological activity of fixed antibodies. At the same time, its corresponding sensor interface can be quickly activated, and its corresponding repeatability can be improved [25]. Relevant scientists in the United States have prepared a biosensor at the level of deoxyribonucleotide biological detection, which mainly uses the irregular edge characteristics of the deoxyribonucleotide to provide high-density active edge sites, so as to expand the detection range [26]. At the research level of the biomedical teaching experiment effect evaluation system, the main system technology includes the computer network and its distributed database management system. At the design level of the biomedical teaching effect evaluation system, a large number of scientists and research institutions have studied and analyzed it, and its main experimental effect is also based on biosensor preparation technology [27].

\section{Preparation of Biosensor and Design of Biomedical Experiment Teaching Evaluation System}

This section mainly analyzes and studies the preparation technology and optimization technology of the graphene biosensor. At the same time, this section will also give the design idea of the corresponding biomedical experiment teaching evaluation system. The corresponding principle block diagram is shown in Figure 1. As can be seen from Figure 1, at the level of biosensor preparation, this paper mainly prepares the electrochemical sensor applied to biomedical experimental teaching based on graphene, optimizes the detection sensitivity and detection range of the graphene electrochemical sensor based on the corresponding experimental conditions, and improves its corresponding stability and reusability. In the aspect of electrochemical activity of biosensors, this paper innovatively uses the electrochemical impedance method to detect the electrochemical activity, so as to accurately evaluate the key characteristics of biosensors. At the design level of the corresponding biomedical experiment teaching evaluation system, the biomedical experiment teaching effect evaluation system is mainly constructed based on the information network.

3.1. Analysis and Study on Preparation Technology of Graphene Biosensor. In this section, the corresponding biosensor in the biomedical experimental teaching system is 
TABLE 1: Experimental reagents and equipment for graphene biosensor preparation.

\begin{tabular}{|c|c|c|c|}
\hline $\begin{array}{l}\text { Serial number of } \\
\text { experimental equipment }\end{array}$ & Name of experimental equipment & Serial number of test reagent & Name of experimental reagent \\
\hline 1 & Ultrasonic cleaning machine & 1 & Mechanical stripping graphene \\
\hline 2 & Electron microscope & 2 & Polyvinylpyrrolidone \\
\hline 3 & Centrifuge & 3 & Secondary distilled water \\
\hline 4 & Balance & 4 & Sodium hydroxide \\
\hline 5 & Precision $\mathrm{pH}$ meter & 5 & $\mathrm{H}_{3} \mathrm{PO}_{4}$ \\
\hline 6 & Laser spectrometer & 6 & \\
\hline 7 & Electrochemical workbench & & \\
\hline
\end{tabular}

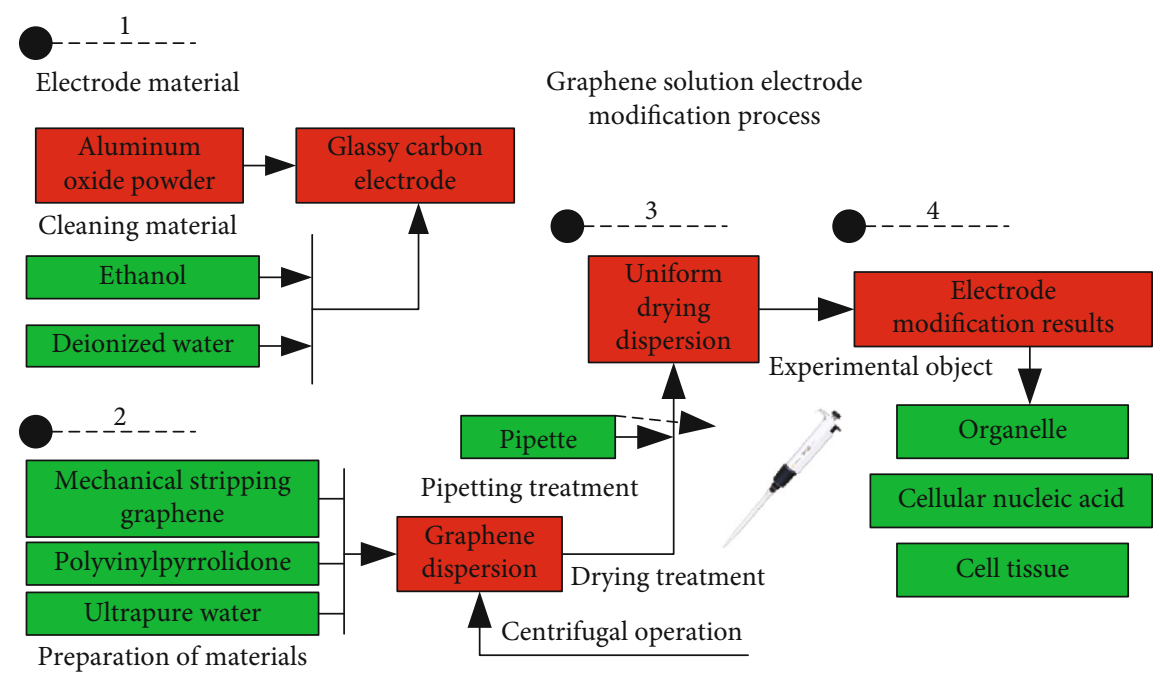

FIgURE 2: Principle block diagram of graphene solution electrode modification process.

designed based on graphene. Graphene and polyvinylpyrrolidone are used as the electrode materials of the biosensor in the preparation process, and the electrochemical sensitivity of the sensor prepared in this paper is optimized based on the characteristics of this material. The water used in the actual preparation process in this paper is distilled water. The corresponding experimental reagents and equipment used are shown in Table 1.

The above conditions are necessary for the preparation of the graphene biosensor. Based on the above experimental reagents and experimental instruments, the key part of the preparation of the graphene biosensor is the graphene solution electrode modification process. The corresponding process principle block diagram is shown in Figure 2. It can be seen from Figure 2 that the graphene preparation process is mainly divided into four key steps. Each corresponding step has its corresponding key technology. The first step is mainly cleaning, the corresponding second step is mainly centrifugation, the corresponding third step is mainly drying treatment technology, and the corresponding fourth step is mainly modification. From Figure 2, it can be seen that the corresponding preparation technical steps are as follows.

Step 1: continuously modify the glassy carbon electrode with aluminum oxide powder, and continuously clean the glassy carbon electrode with ethanol and deionized water. After cleaning, blow dry with nitrogen.
Step 2: graphene dispersion is prepared based on mechanical stripping of graphene, polyvinylpyrrolidone, and corresponding ultrapure water, and uniform dispersion is obtained by centrifugation based on this dispersion.

Step 3: based on the pipette, place the corresponding dispersion on the surface of the glassy carbon electrode for drying treatment.

Step 4: the electrode modification results of the graphene solution were obtained.

In order to further optimize the electrochemical performance of the graphene biosensor, cyclic voltammetry is used to verify and optimize the selection of the graphene surface area. The core significance of the optimization algorithm is to characterize the corresponding electron mobility on the electrode surface through the oxidation current on the glassy carbon electrode. The electrochemical sensitivity of the graphene biosensor is positively represented based on electron mobility. In this paper, when cyclic voltammetry is used, the linear voltage characteristic is used to continuously scan from high voltage to low voltage and then repeatedly scan back. The corresponding scanning function curve is shown in Figure 3. It can be seen from the figure that the corresponding detection curve includes two parts: first, when the potential sweeps to the cathode, the detected substance will get electrons, so that the reduction peak can be detected; second, when the corresponding potential sweeps to the 


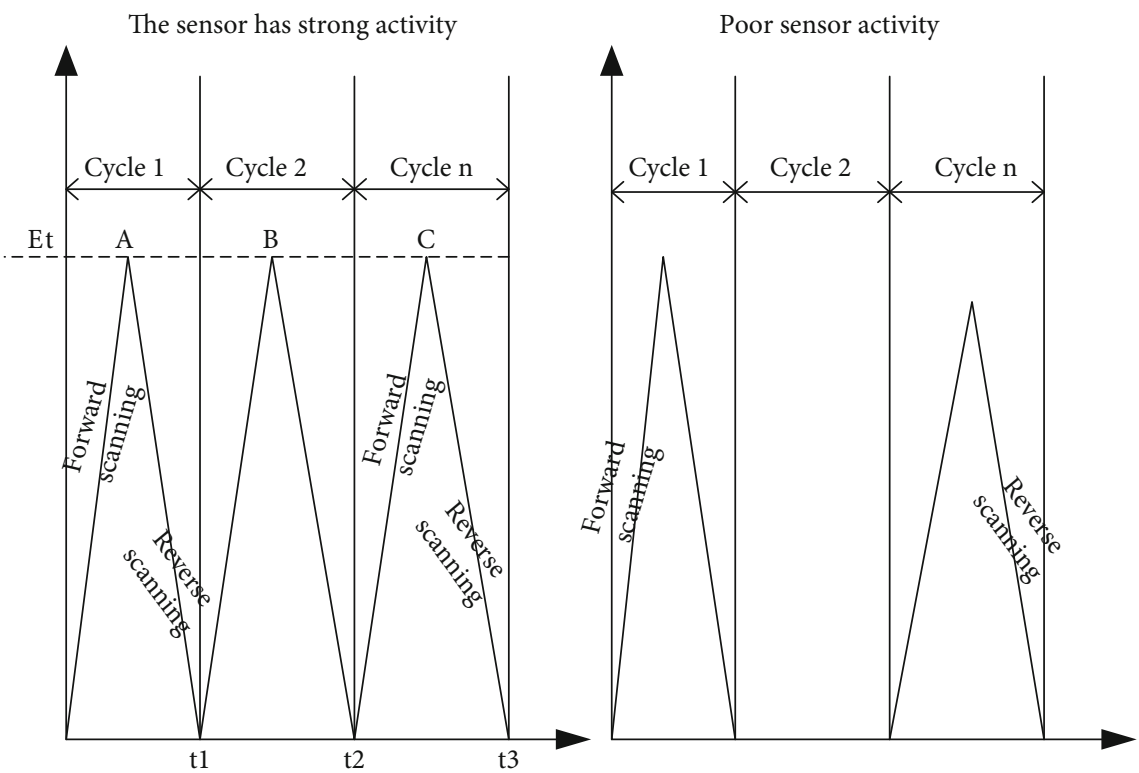

FIGURE 3: Scanning function curve of cyclic voltammetry.

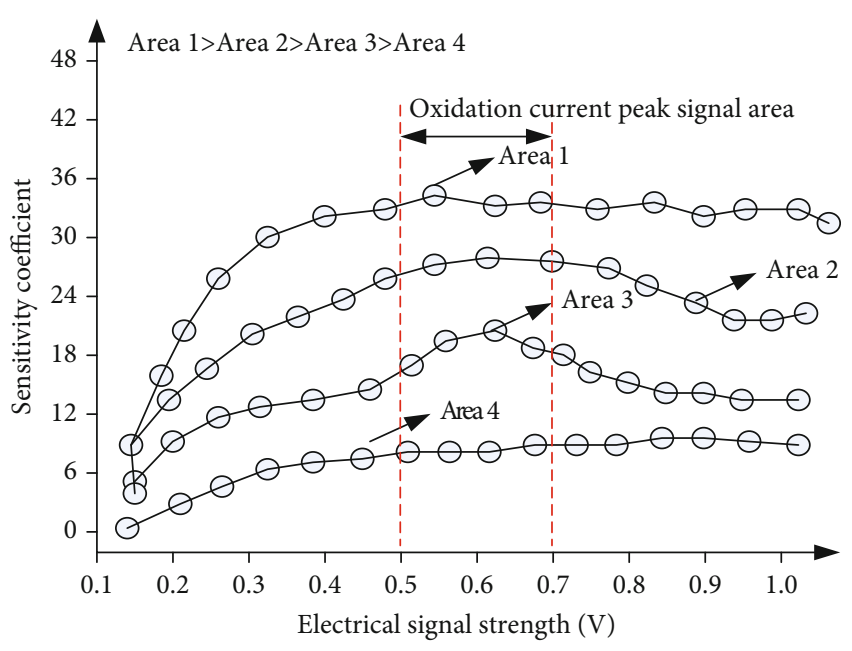

FIGURE 4: Electrochemical sensitivity curves of graphene biosensors with different surface areas.

anode, an oxidation peak will appear. Based on this repeated scanning, the process of continuous gain and loss of electrons is realized. At this time, when the activity of the corresponding detected substance is high, the corresponding two peaks are symmetrical and the corresponding amplitude difference is small. When the activity of the corresponding substance is poor, only one corresponding oxidation or reduction reaction will occur; thus, there is only one corresponding oxidation peak and reduction peak. Therefore, based on the above principle, the performance of the selected biosensor can be sensitively reflected.

In order to further optimize the electrochemical performance of the graphene biosensor, cyclic voltammetry is used to verify and optimize the selection of the graphene surface area. The core significance of the optimization algorithm is to characterize the corresponding electron mobility on the electrode surface through the oxidation current on the glassy carbon electrode; the electrochemical sensitivity of graphene biosensor is positively represented based on electron mobility. The corresponding electrochemical sensitivity curves of graphene biosensors with different surface areas are shown in Figure 4. It can be seen from Figure 4 that selecting a larger graphene surface area is conducive to improving the electrochemical sensitivity of the biosensor and can give full play to the good crystal structure and catalytic performance of graphene to the greatest extent.

3.2. Design and Analysis of Biomedical Experiment Teaching Evaluation System. The biomedical teaching evaluation system designed in this paper is mainly to better evaluate biological experimental teaching and reflect the practical role of biosensors in biomedical experimental teaching. The system designed in this paper includes five modules: sensor data processing and analysis module, experimental information management module, student experimental feedback module, teacher classroom feedback module, and overall evaluation module. The principle framework of the corresponding biomedical experimental teaching evaluation system is shown in Figure 5.

In the corresponding sensor data processing module, the graphene biosensor prepared above is mainly used as the medium for students to perceive biological characteristics such as microbiological organelles, so as to help students better understand the conceptual elements in biomedical experiments and corresponding biomedical phenomena. By transforming the biological information sensed by the biosensor into physical signals such as electrical signals and sending them to the computer for processing and analysis, students can more comprehensively and intuitively understand the relevant experimental steps and implementation phenomena, so as to deeply quantify the experimental results, so as to further improve the effect of biomedical experimental teaching. 


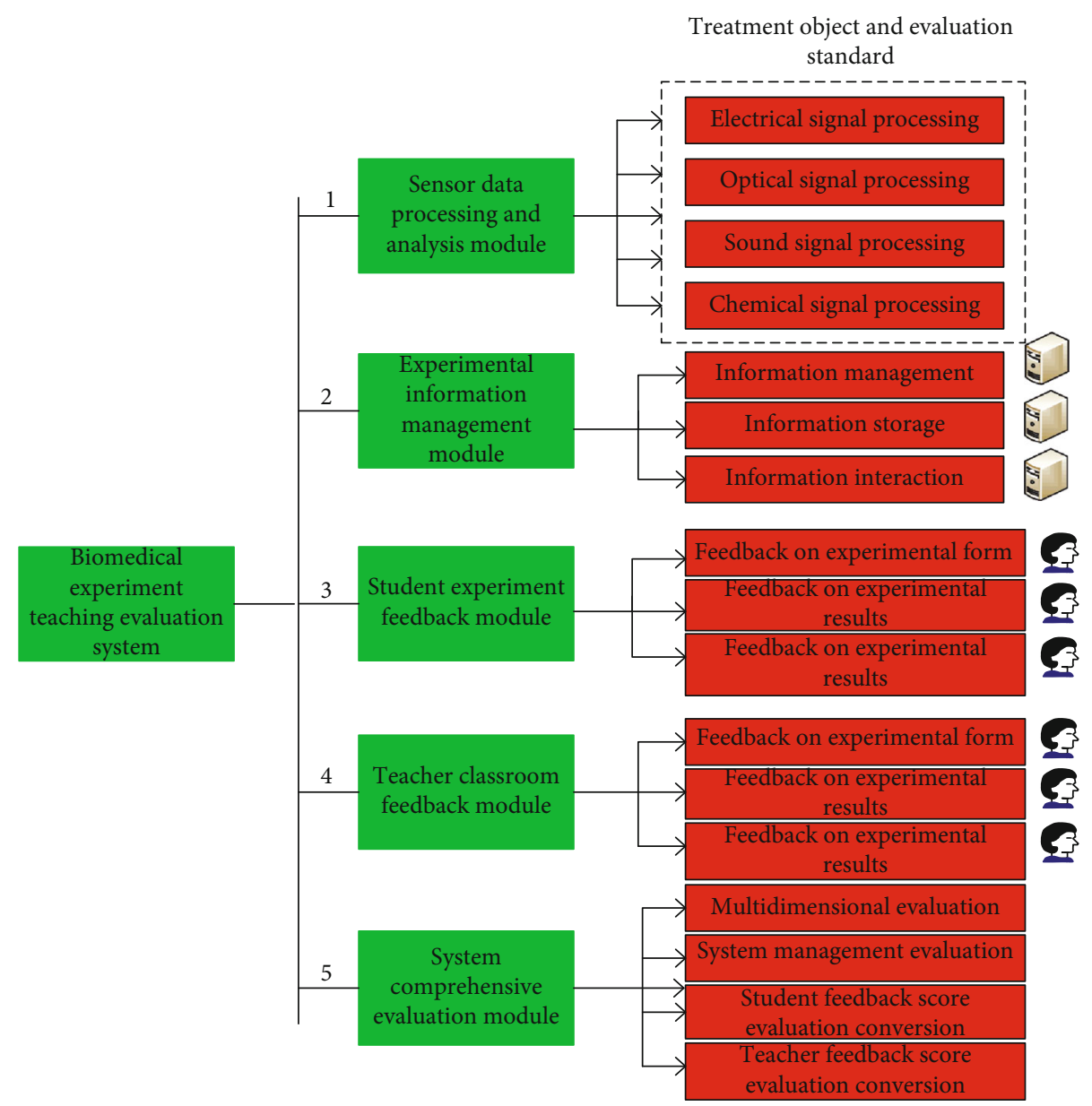

Figure 5: Principle frame diagram of biomedical experiment teaching evaluation system.

The corresponding information system management level mainly adopts the $\mathrm{C} / \mathrm{S}$ architecture, the corresponding physical architecture adopts the LAN form, the corresponding database level adopts the dynamic database technology, and the network communication protocol mainly adopts the TCP/IP protocol. The information system management module is mainly used to comprehensively manage the biological data collected by biosensors, as well as various biomedical experiments and the information of students and teachers. At the same time, the information system management module also needs to comprehensively deal with the evaluation of students and teachers on biomedical experiments and form final opinions.

The student experiment feedback module is mainly used to provide an interface for students to timely feed back the current biomedical experiment teaching effect. It is mainly used to store and analyze students' opinions and evaluation on the experiment and also test the current experimental situation of students, Thus, students' evaluation results of biomedical experimental teaching are given at the subjective and objective levels and finally outputted to the evaluation module for integration with teachers' evaluation opinions.

The teacher classroom feedback module mainly provides a subjective teaching effect evaluation interface for teachers. It records, stores, and analyzes the teachers' subjective eval- uation of this biomedical teaching experiment and finally converts it into a score calculated according to a certain proportion as the evaluation index of the current biomedical teaching experiment at the teacher level.

The overall evaluation module mainly integrates the contents of the student experimental feedback module and the teacher classroom feedback module for comprehensive processing and analysis. At the same time, the results of the comprehensive processing and analysis are displayed in the form of final scores as the evaluation results of the current biomedical experimental teaching.

In the security level of the whole biomedical teaching evaluation system, this paper mainly controls the operation of the system based on user authentication and key and realizes the safe operation of the system by designing hierarchical permissions for the application system.

\section{Experimental Verification and Analysis}

In order to verify the advantages of the graphene biosensor prepared in this paper compared with the traditional biosensor in biomedical experiments, and to verify the practicability of the biomedical evaluation system proposed in this paper, this section will analyze and discuss the detection experiment and experimental effect evaluation based on tryptophan. 


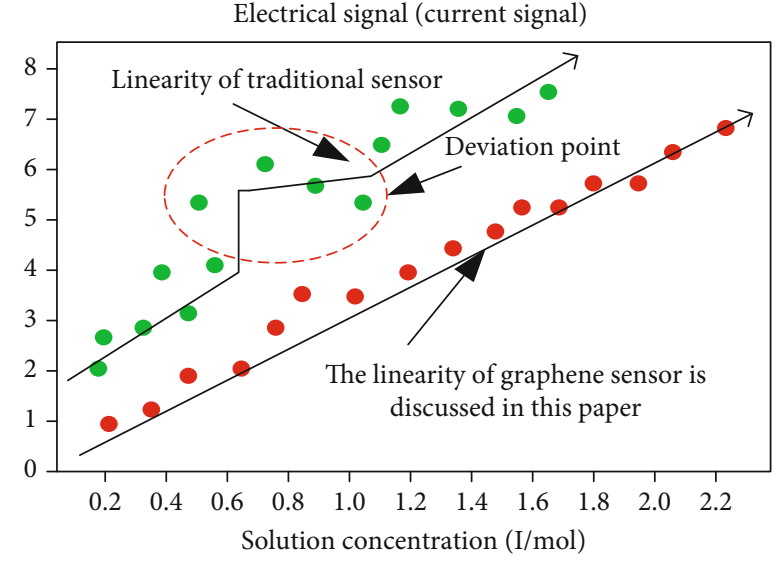

(a)

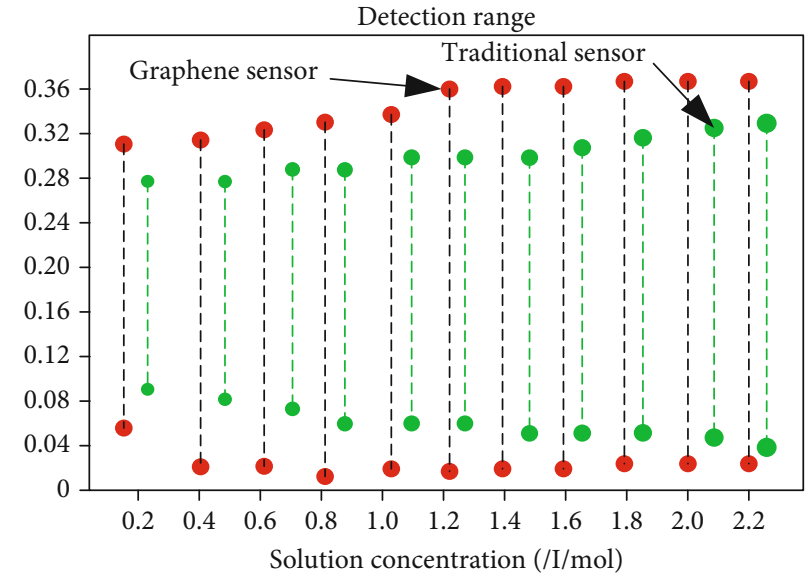

(b)

FIgURE 6: (a) Detection linearity curve; (b) monitoring range curve.

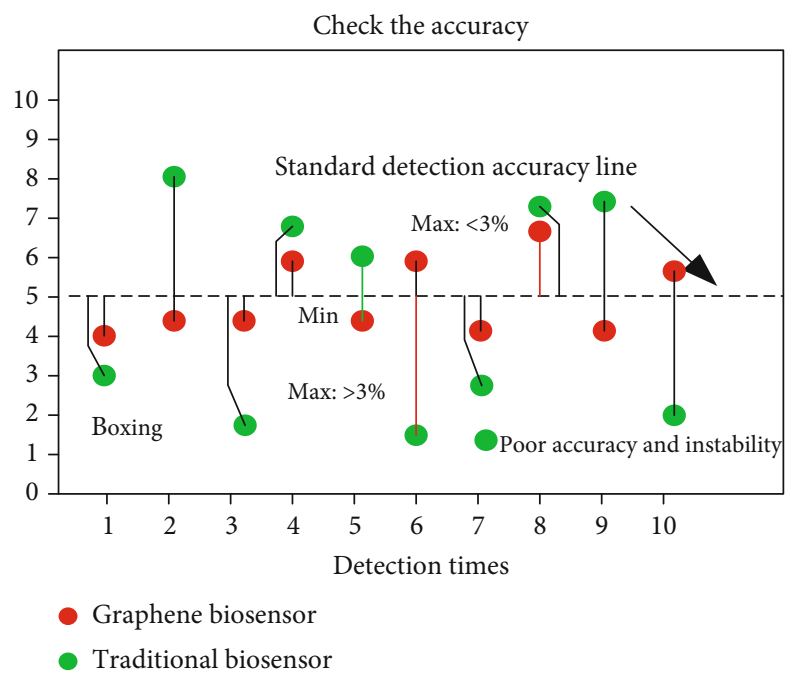

FIGURE 7: Detection curve of reproducibility characteristics of biosensor.

The superiority of the sensor is mainly verified by analyzing its corresponding linear detection range and corresponding detection limit. Based on this, experiments are carried out on tryptophan detection solutions with different concentrations. The corresponding experimental results are shown in Figures 6(a) and 6(b). It can be seen from the figure that the graphene biosensor used in this paper has better detection linearity than the traditional biosensor, and its corresponding detection range is also relatively wide.

In order to further verify the reproducibility advantages of the graphene biosensor proposed in this paper, the same modified electrode is used to measure the solution of the same concentration for 10 times, and the corresponding biosensor current signal deviation is recorded. The corresponding experimental result curve is shown in Figure 7. It can be seen from the figure that the graphene biosensor proposed in this paper has better repeatability than the traditional biosensor, and the corresponding deviation is less than $3 \%$.

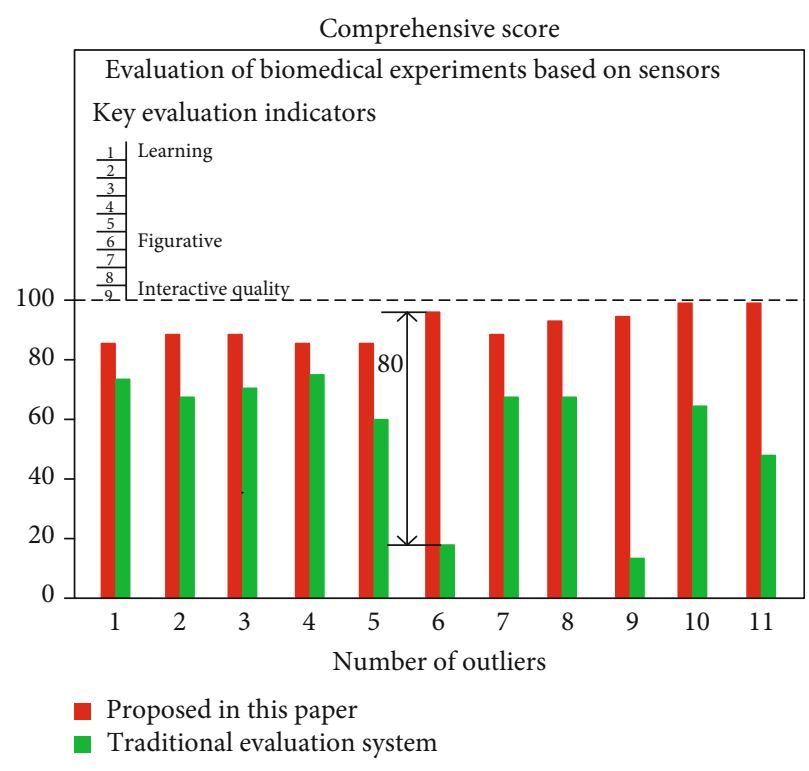

FIGURE 8: Effect curve of biomedical experiment evaluation.

In order to reflect the advantages of this biomedical teaching evaluation system, the teaching experiment evaluation is carried out based on the tryptophan experiment, and the corresponding evaluation index is mainly the comprehensive evaluation score. The evaluation effect of biomedical experiments based on the two biosensors is shown in Figure 8. It can be seen from the figure that the evaluation effect of biomedical experiments based on graphene biosensors is due to the similar experiments carried out by traditional biosensors.

According to the above experimental results, the sensorbased biomedical experiment is analyzed. The main analysis contents include the following: description of the modification characteristics of biosensor electrode, analysis of the advantages of the electrochemical characteristics of graphene biosensor, the impact of $\mathrm{pH}$ on the biomedical experiment, and the impact of the performance of biosensor on the effect of biomedical teaching experiment. For the 
TABle 2: Optimization and improvement of graphene biosensor and biomedical teaching experiment.

\begin{tabular}{lc}
\hline Optimization part & Optimization level details \\
\hline $\begin{array}{l}\text { Optimization of electrode } \\
\text { of graphene biosensor } \\
\text { modification parameters }\end{array}$ \\
$\begin{array}{cc}\text { Optimization of material deposition } \\
\text { pH optimization } \\
\text { Polarization voltage optimization } \\
\text { Selection and optimization of } \\
\text { biosensor } \\
\text { biomedical teaching } \\
\text { experiment }\end{array}$ \\
$\begin{array}{c}\text { Optimization of teacher-student } \\
\text { interaction }\end{array}$ \\
\end{tabular}

electrode modification characteristics of biosensors, compared with ordinary biosensors, the large-area graphene biosensor proposed in this paper can better adsorb and fix biological macromolecules in biomedical experiments, so as to promote the sensitivity of biomedical experimental detection and enable students to obtain more real detection results; at the same time, it also provides a guarantee for the follow-up experimental conclusions. In terms of the advantages of the electrochemical characteristics of the graphene biosensor, the above experiments further prove that the electrochemical performance detection based on cyclic voltammetry has higher accuracy; at the same time, the experimental results under cyclic voltammetry further prove that the mechanical stripping graphene corresponding to the graphene biosensor proposed in this paper has more advantages than the corresponding crystal structure of the traditional biosensor (detection of tryptophan and such biological experiments), and its corresponding catalytic performance is better. In terms of the impact of $\mathrm{pH}$ on biomedical experiments, this paper does not give the experimental results in the experimental part, but it is important to analyze the $\mathrm{pH}$ from the experimental process, but this paper controls the $\mathrm{pH}$ of the same experiment. In terms of the performance of biosensors on the effect of biomedical teaching experiments, good biosensors play an important role in improving the quality of biomedical teaching. Through the introduction of biosensors with high precision, high reproducibility, and fast response speed, students' interest in learning can be significantly improved, and the reliability of experiments and the accuracy of experimental conclusions can be increased; at the same time, it can also provide good teaching support for experimental teachers and improve classroom quality.

Based on the above analysis, the graphene biosensor is optimized, and the biomedical teaching experiment based on the biosensor is improved, so as to further improve the experimental teaching effect. The corresponding optimization direction is shown in Table 2. The optimization of electrode parameters in the corresponding table mainly considers the optimization of electrode modification parameters to obtain high sensitivity. The optimal solution of this parameter depends on the experimental results and analysis. The material deposition parameters will seriously affect the corresponding polymerization state, polymerization morphology, and corresponding polymerization density on the surface of glassy carbon electrode, which has an important restriction on the sensitivity of the sensor. Therefore, at the level of material deposition parameter optimization, based on cyclic voltammetry, it is found that when the corresponding electrode scanning is about 20 cycles, the corresponding biosensor has the highest sensitivity. At the corresponding $\mathrm{pH}$ optimization level, when the corresponding $\mathrm{pH}$ is neutral or near neutral, the corresponding biosensor sensitivity is the highest. The corresponding experimental results also show that when the corresponding electrolyte is too acidic or alkaline, the corresponding biosensor sensitivity will be greatly reduced. At the corresponding polarization voltage level, the corresponding polarization voltage will seriously affect the detection sensitivity of the biosensor. The polarization voltage corresponding to different electrolytes needs to be obtained through experiments. In the experiment, it is found that the corresponding biosensor has the highest sensitivity when the polarization voltage is maintained at about $0.1 \mathrm{~V}$. When the corresponding polarization voltage is higher than $0.1 \mathrm{~V}$, the corresponding sensor sensitivity will be greatly reduced, but the polarization voltage should not be too small, which will affect the adequacy of the reduction reaction of the experiment. In the aspect of biomedical experimental teaching optimization, it is necessary to emphasize the selection and optimization of biosensors in the experiment, enhance the interaction between teachers and students in the experimental process, and optimize the biomedical experimental teaching evaluation system.

\section{Conclusion}

This paper mainly analyzes the research status of the biosensor and its biomedical experimental teaching evaluation system and summarizes its corresponding existing problems. In view of the current characteristics of low precision, poor repeatability, and unsystematic evaluation system of biomedical experimental teaching, this paper mainly prepares the electrochemical sensor for biomedical experimental teaching based on graphene and optimizes the detection sensitivity and detection range of graphene electrochemical sensor based on the corresponding experimental conditions; at the same time, its corresponding stability and reusability are improved. At the level of electrochemical activity of biosensors, this paper innovatively uses the electric AC impedance method to detect the electrochemical activity, so as to accurately evaluate the key characteristics of biosensors. Based on the preparation of biosensors and the results of biological experiments, this paper will design a biomedical experiment teaching effect evaluation system based on the network and realize the basic functions of teacher-student interaction,teaching effect evaluation, sensor performance evaluation and so on. Based on the above, the electrochemical sensor based on graphene and conductive polymer solution is actually prepared in this paper. At the same time, the electrocatalysis experiment is carried out based on the 
sensor, and the experimental teaching effect is systematically evaluated. The experimental results show that the biosensor proposed in this paper has the advantages of high sensitivity, simple preparation, and high availability; at the same time, it also further verifies that the biomedical experimental teaching effect evaluation system proposed in this paper has good evaluation effect and can provide accurate reference for the evaluation of biological experimental teaching effect. In the follow-up research, this paper will focus on the accuracy of the graphene sensor in biological detection and its corresponding biomedical teaching effect in other harsh environments. At the same time, this paper will continue to study the application of other nanomaterials in the preparation of biosensors and continuously improve its accuracy and repeatability.

\section{Data Availability}

The data used to support the findings of this study are available from the corresponding author upon request.

\section{Conflicts of Interest}

The author declares that they have no conflicts of interest.

\section{Funding}

This work was supported by the 2021 Education and Teaching Reform Research and Practice Project of Xuchang University (general project) "Project Name: Application of TBL Teaching Method and Experiential Teaching Method in Physiology Teaching in Colleges and Universities" (No.: XCU2021-YB-060).

\section{References}

[1] H. Zhao, X. Ji, B. Wang et al., "An ultra-sensitive acetylcholinesterase biosensor based on reduced graphene oxide-Au nanoparticles- $\beta$-cyclodextrin/Prussian blue-chitosan nanocomposites for organophosphorus pesticides detection," Biosensors \& Bioelectronics, vol. 65, no. 4, pp. 23-30, 2015.

[2] E. Mehmeti, D. M. Stankovi, and S. Chaiyo, "Wiring of glucose oxidase with graphene nanoribbons: an electrochemical third generation glucose biosensor," Microchimica Acta, vol. 184, no. 4, pp. 1127-1134, 2017.

[3] S. Han, T. Du, J. Hui, and X. Wang, "Synergistic effect of pyrroloquinoline quinone and graphene nano-interface for facile fabrication of sensitive NADH biosensor," Biosensors \& Bioelectronics, vol. 89, no. 1, pp. 422-429, 2017.

[4] S. Beyranvand, M. F. Gholami, A. D. Tehrani, J. P. Rabe, and M. Adeli, "Construction and evaluation of a self-calibrating multiresponse and multifunctional graphene biosensor," Langmuir, vol. 35, no. 32, pp. 10461-10474, 2019.

[5] C. Wang, Y. Li, Y. Zhu et al., "High-kappa solid-gate transistor configured graphene biosensor with fully integrated structure and enhanced sensitivity," Advanced Functional Materials, vol. 26, no. 47, pp. 8575-8575, 2016.

[6] L. Klukova, J. Filip, and T. Belick, "Graphene oxide-based electrochemical label-free detection of glycoproteins down to aM level using a lectin biosensor," Analyst, vol. 141, no. 14, pp. 4278-4282, 2018.
[7] T. Hu, L. Zhang, W. Wen, X. Zhang, and S. Wang, "Enzyme catalytic amplification of miRNA-155 detection with graphene quantum dot-based electrochemical biosensor," Biosensors \& Bioelectronics, vol. 77, no. 4, pp. 451-456, 2016.

[8] J. B. Maurya, Y. K. Prajapati, V. Singh, J. P. Saini, R. Tripathi, and V. Singh, "Improved performance of the surface plasmon resonance biosensor based on graphene or $\mathrm{MoS}_{2}$ using silicon," Optics Communications, vol. 359, no. 4, pp. 426-434, 2016.

[9] M. S. Khan, S. K. Misra, Z. Wang et al., "Paper-based analytical biosensor chip designed from graphene-nanoplatelet-amphiphilic-diblock-co-polymer composite for cortisol detection in human saliva," Analytical Chemistry, vol. 89, no. 3, pp. $2107-$ 2115, 2017.

[10] H. Zhang, Y. Wang, D. Zhao et al., "Universal fluorescence biosensor platform based on graphene quantum dots and pyrene-functionalized molecular beacons for detection of microRNAs," ACS Applied Materials \& Interfaces, vol. 7, no. 30, pp. 16152-16156, 2015.

[11] T. C. Lin, Y. S. Li, W. H. Chiang, Z. Pei, and W. H. Chiang, "A high sensitivity field effect transistor biosensor for methylene blue detection utilize graphene oxide nanoribbon," Biosensors \& Bioelectronics, vol. 89, no. 1, pp. 511-517, 2017.

[12] S. Eissa, M. Zourob, and M. Zourob, "In vitro selection of DNA aptamers targeting $\beta$-lactoglobulin and their integration in graphene-based biosensor for the detection of milk allergen," Biosensors \& Bioelectronics, vol. 91, no. 4, pp. 169-174, 2017.

[13] Q. Wu, Y. Sun, P. Ma et al., "Gold nanostar-enhanced surface plasmon resonance biosensor based on carboxylfunctionalized graphene oxide," Analytica Chimica Acta, vol. 913, no. 4, pp. 137-144, 2016.

[14] M. S. Rahman, M. R. Hasan, K. A. Rikta, M. S. Anower, and K. A. Rikta, "A novel graphene coated surface plasmon resonance biosensor with tungsten disulfide $\left(\mathrm{WS}_{2}\right)$ for sensing DNA hybridization," Optical Materials, vol. 75, no. 6, pp. 567-573, 2018.

[15] M. S. Rahman, M. S. Anower, M. R. Hasan, M. B. Hossain, M. I. Haque, and M. R. Hasan, "Design and numerical analysis of highly sensitive $\mathrm{Au}-\mathrm{MoS}_{2}$-graphene based hybrid surface plasmon resonance biosensor," Optics Communications, vol. 396, no. 5, pp. 36-43, 2017.

[16] J. Sethi, M. V. Bulck, A. Suhail, M. Safarzadeh, A. Perez-Castillo, and G. Pan, "Correction to: a label-free biosensor based on graphene and reduced graphene oxide dual-layer for electrochemical determination of beta-amyloid biomarkers," Microchimica Acta, vol. 187, no. 6, pp. 12-16, 2020.

[17] A. A. Ensafi, M. Sohrabi, M. Jafari-Asl, B. Rezaei, and M. Jafari-Asl, "Selective and sensitive furazolidone biosensor based on DNA-modified $\mathrm{TiO}_{2}$-reduced graphene oxide," Applied Surface Science, vol. 356, no. 11, pp. 301-307, 2015.

[18] K. Nakama, M. Sedki, A. Mulchandani, and A. Mulchandani, "Label-free chemiresistor biosensor based on reduced graphene oxide and M13 bacteriophage for detection of coliforms," Analytica Chimica Acta, vol. 1150, no. 15, article 338232, 2021.

[19] Y. H. Nien, T. Y. Su, C. S. Ho et al., "The analysis of potentiometric flexible arrayed urea biosensor modified by graphene oxide and $\gamma$ - $\mathrm{Fe}_{2} \mathrm{O}_{3}$ nanoparticles," IEEE Transactions on Electron Devices, vol. 67, no. 11, pp. 5104-5110, 2020.

[20] D. Chauhan, Pooja, V. Nirbhaya et al., "Nanostructured transition metal chalcogenide embedded on reduced graphene 
oxide based highly efficient biosensor for cardiovascular disease detection," Microchemical Journal, vol. 155, no. 3, article 104697, 2020.

[21] J. Dong, M. Sang, S. Wang et al., "Ultrasensitive label-free biosensor based on the graphene-oxide-coated-U-bent longperiod fiber grating inscribed in a two-mode fiber," Journal of Lightwave Technology, vol. 39, no. 12, pp. 4013-4019, 2021.

[22] M. Devaraj, S. Rajendran, J. N. Jebaranjitham et al., "Horseradish peroxidase-immobilized graphene oxide-chitosan gold nanocomposites as highly sensitive electrochemical biosensor for detection of hydrogen peroxide," Journal of the Electrochemical Society, vol. 167, no. 14, article 147517, 2020.

[23] F. N. Ajeel, A. M. Khudhair, M. H. Mohammed, K. M. Mahdi, and M. H. Mohammed, "DFT investigation of graphene nanoribbon as a potential nanobiosensor for tyrosine amino acid," Russian Journal of Physical Chemistry A, vol. 93, no. 4, pp. 778-785, 2019.

[24] M. Zhao, L. Bai, W. Cheng, X. Duan, H. Wu, and S. Ding, "Monolayer rubrene functionalized graphene-based eletrochemiluminescence biosensor for serum cystatin $\mathrm{C}$ detection with immunorecognition-induced 3D DNA machine," Biosensors and Bioelectronics, vol. 127, no. 55, pp. 126-134, 2019.

[25] H. Borah, S. Gogoi, S. Kalita, P. Puzari, and S. Kalita, “A broad spectrum amperometric pesticide biosensor based on glutathione S-transferase immobilized on graphene oxide-gelatin matrix," Journal of Electroanalytical Chemistry, vol. 828, no. 4, pp. 116-123, 2018.

[26] S. Proa-Coronado, J. R. Vargas-García, A. Manzo-Robledo et al., "Platinum nanoparticles homogenously decorating multilayered reduced graphene oxide for electrical nanobiosensor applications," Thin Solid Films, vol. 658, no. 7, pp. 54-60, 2018.

[27] J. White, K. Costilow, J. Dotson et al., "Guided-inquiry experiment for teaching the calibration method of standard addition in the analysis of lead with graphite furnace atomic absorption spectroscopy," Journal of Chemical Education, vol. 98, no. 2, pp. 620-625, 2021. 